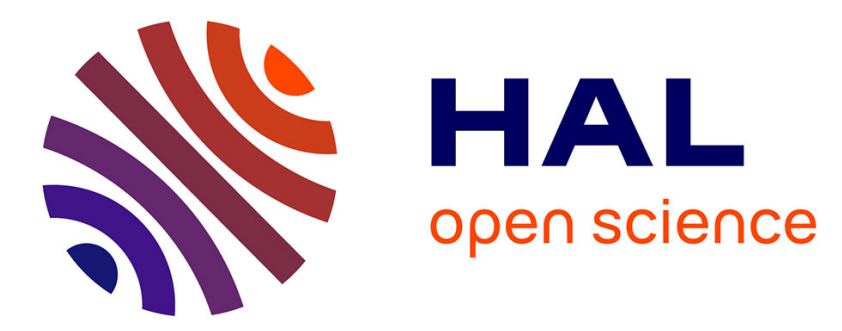

\title{
Multi-scalar analysis of hip implant components using modal decomposition
}

J Grandjean, G. Le Goic, H. Favreliere, Y. Ledoux, S. Samper, F. Formosa, L Devun, T Gradel

\section{- To cite this version:}

J Grandjean, G. Le Goic, H. Favreliere, Y. Ledoux, S. Samper, et al.. Multi-scalar analysis of hip implant components using modal decomposition. Measurement Science and Technology, 2012, 23 (12), pp.125702. 10.1088/0957-0233/23/12/125702 . hal-02136834

\section{HAL Id: hal-02136834 \\ https://hal.science/hal-02136834}

Submitted on 22 May 2019

HAL is a multi-disciplinary open access archive for the deposit and dissemination of scientific research documents, whether they are published or not. The documents may come from teaching and research institutions in France or abroad, or from public or private research centers.
L'archive ouverte pluridisciplinaire HAL, est destinée au dépôt et à la diffusion de documents scientifiques de niveau recherche, publiés ou non, émanant des établissements d'enseignement et de recherche français ou étrangers, des laboratoires publics ou privés. 
Multi-scalar analysis of hip implant components using modal decomposition

This article has been downloaded from IOPscience. Please scroll down to see the full text article.

2012 Meas. Sci. Technol. 23125702

(http://iopscience.iop.org/0957-0233/23/12/125702)

View the table of contents for this issue, or go to the journal homepage for more

Download details:

IP Address: 193.48.131.250

The article was downloaded on 16/11/2012 at 08:45

Please note that terms and conditions apply. 


\title{
Multi-scalar analysis of hip implant components using modal decomposition
}

\author{
J Grandjean ${ }^{1,2}$, G Le Goic ${ }^{1}$, H Favreliere ${ }^{1}$, Y Ledoux ${ }^{2}$, S Samper ${ }^{1}$, \\ F Formosa ${ }^{1}, \mathbf{L}$ Devun ${ }^{3}$ and T Gradel ${ }^{3}$ \\ ${ }^{1}$ SYMME Laboratory, Université de Savoie, BP 80439, 74944 Annecy-le-Vieux Cedex, France \\ ${ }^{2}$ Université de Bordeaux, I2M, UMR 5295, F-33400 Talence, France \\ ${ }^{3}$ TURAL Society, Department of Researches, Marignier, France \\ E-mail: Julien.Grandjean@univ-savoie.fr
}

Received 19 March 2012, in final form 21 September 2012

Published 15 November 2012

Online at stacks.iop.org/MST/23/125702

\begin{abstract}
This paper presents a metrological analysis of hip prosthesis components. When changing ceramic prostheses, the surgeon sometimes finds traces of alloy lying in the insert or the femoral head. These traces can be thin and accurate or as a wide band. From the measurements made on the contact areas of hip prosthesis components, we analyse these phenomena by highlighting the defects of form, waviness and roughness of these surfaces using a novel geometric parameterization (namely modal parameterization). The aim of this work is to isolate these defects to characterize the prostheses components. We show that this parameterization allows a multi-scale analysis of surfaces regardless of the type of wear of the prosthesis, and that the results offer some relevant explanations to the analysis of visible damage on the prostheses. In a later study, we are going to analyse the defects influence on the alteration of the performance of hip prostheses.
\end{abstract}

Keywords: surface analysis, surface roughness, total hip arthroplasty, modal decomposition, surface metrology

(Some figures may appear in colour only in the online journal)

\section{Introduction}

Total hip arthroplasty is one of the most frequently performed procedures in orthopaedic surgery (150000 procedures in France annually). Today, it allows numerous people suffering from degenerative disease or acute inflammation to walk without pain. From a mechanical point of view, this prosthesis is a spherical connection that functions in the same manner as the replaced joint. While replacing ceramic prostheses, a surgeon will sometimes note wear marks or metal alloy incrustations on the spherical part of the explants. These incrustations can be thin and very localized or in the form of a wide band. They can cause the prostheses to deteriorate prematurely or result in other minor malfunctions (such as noise).
This paper presents a metrological study of these phenomena to provide some principles for geometrically analysing spherical surface defects.

Geometric measurements are typically decomposed into six orders of defects that are defined according to technological and mathematical criteria (International Organization for Standardization 1998). The first orders, size and position, are processed according to mathematical models based on the theory of mechanisms. In this paper, we concentrate on form, waviness and surface roughness defects. The surfaces studied are the contact surfaces between joint components associated with the ball-and-socket connection, which has a nominally spherical form. Using scatter plots that are derived from measurements of the examined surface and obtained by extended-field confocal microscopy, modal parameterization 


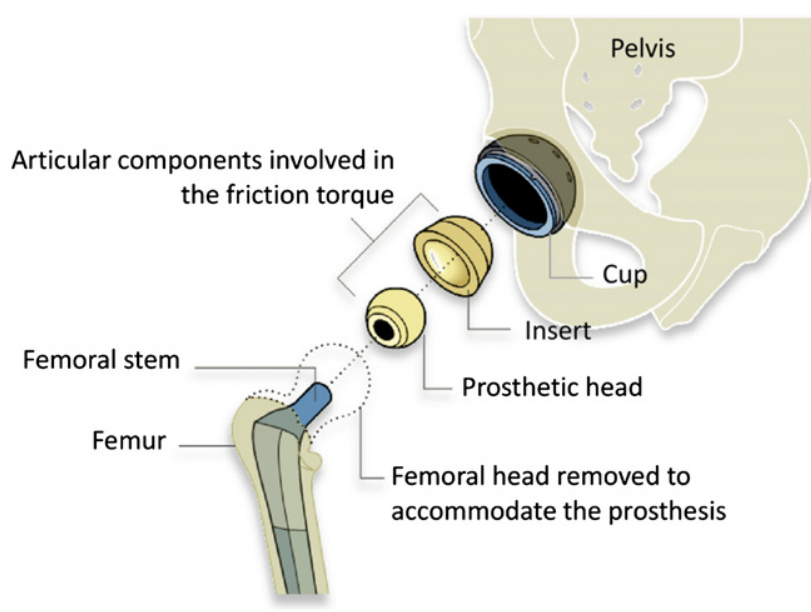

(a) Components of the prosthesis

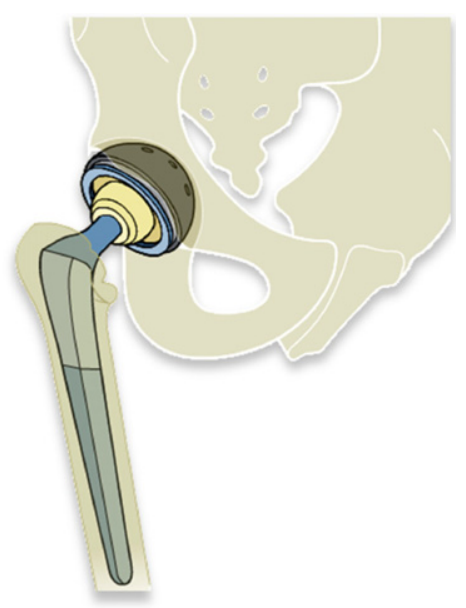

(b) Assembled prosthesis

Figure 1. Total hip implant (THI).

is used to exhaustively decompose the examined surface into a vector basis without a priori knowledge of the surface's form. This decomposition sorts the defects by order of frequency and increasing complexity (Le Goï et al 2011). This paper shows that this technique can be used to filter the form, waviness and surface roughness components, regardless of the type or amount of wear of the examined part.

A subsequent study will analyse the assemblies as a function of their scale and conditions of use and the influence of geometric defects observed on prosthetic joint components on the function and performance impairment of these assemblies.

\section{Analysis of a hip prosthesis using the modal method}

\subsection{Total hip implant (THI)}

2.1.1. Introduction. The hip joint connects the joint cavity of the pelvis (the acetabulum) to the femoral head (hemispherical end of the thigh bone). A THI (figure 1) consists of a cup, femoral stem and prosthetic head. The cup is the upper component of the THI and consists of a metal hemisphere (titanium or cobalt-chrome) set into the acetabulum. A polyethylene, metal or ceramic lining, referred to as the insert, is lodged into the cup. The femoral stem is a metal component (titanium, steel, or cobalt-chrome) that is inserted into the upper end of the femur. This stem supports the prosthetic head (ceramic or cobalt-chrome), which is attached to the neck of the femoral stem and fits into the insert.

The materials most commonly used to reduce the friction between the prosthetic head and insert are combined in a metal-on-polyethylene wear couple. However, polyethylene degradation is significant and provokes the phenomenon of osteolysis, wherein the organism seeks to remove foreign particles. This reaction weakens the bones and can cause the prosthesis to loosen. Polyethylene improvements, such as cross-linked polyethylene, increase its resistance to wear and thus limit the occurrence of osteolysis. However, technological improvements have led to the development of new friction-reducing material combinations, such as ceramicon-ceramic wear couples, which today compete with metalon-polyethylene wear couples. The ceramic-on-ceramic wear couple was developed in 1970 but its use was limited due to the fragility of the ceramic, which can lead to fractures in the implants. This problem was partly solved by the development of new ceramics in 1995. The risk of fracture is very limited with these new materials, with an estimated risk between $0.005 \%$ and $0.02 \%$ for alumina ceramic and an even lower risk for composite alumina-zirconium ceramics (Chevillotte et al 2008, Hannouche et al 2003).

2.1.2. Squeaking. In some cases, implanted ceramic THIs emit a noise, often referred to as squeaking, when the patient moves. According to Swanson et al (2010) this painless phenomenon affects $1 \%$ of THI and constitutes a real inconvenience to patients in $25 \%$ of those cases. For more severe cases, a surgical procedure known as revision is necessary to replace the implant.

The literature shows that numerous factors can provoke squeaking, such as poor implantation of a prosthetic component, the off-centred loading of the prosthesis (edge loading), the presence of foreign particles (also referred to as third-body particles) between the two spherical parts of the joint, or poor lubrication related, for example, to the patient's pathology (Walter et al 2010).

Restrepo et al (2010) and Jarrett et al (2009) showed that an overhanging acetabular lip can be responsible for the presence of metal particles between the spherical parts, resulting in squeaking. A second type of wear, referred to as stripe wear, is often found on the explants of squeaky prostheses and is characterized by rough areas on the head and insert (Matar et al 2010, Restrepo et al 2008). Taylor et al (2007) and Chevillotte et al (2008) also confirmed that this phenomenon could cause squeaking through testing under in vitro conditions. 
2.1.3. Stripe wear. Since ceramic prostheses were originally developed, rough areas have been found on the head and insert of many explants, disclosing intra-granular fractures and the erosion of rubbing surfaces (Nevelos et al 2000, Sariali et al 2010). This specific type of wear is called stripe wear due to the resulting band of deterioration.

2.1.4. Metallic incrustations. On certain ceramic explants, alloy incrustation marks can be found on the insert or the femoral head. These third-body incrustations may be thin and very localized or in the form of bands that are wider than those caused by stripe wear. These incrustations are likely due to impingement or friction between the ceramic and metal parts of the prosthesis or, if metal deposits are left by the surgeon during the implanting of the prosthesis, due to friction between the tools and ceramic parts.

\subsection{Analysis method}

To provide quantitative information on these different phenomena, which could cause the onset of squeaking, we propose to study two prosthetic heads that show deterioration by metal deposit inclusions. The wear of the first prosthetic head is superficial, whereas the second presents a more advanced stage of deterioration. We believe that these phenomena could potentially modify the state of the prosthesis surface and change the pressure and lubrication conditions of the prosthesis. The conjunction of these phenomena may cause squeaking to occur.

This study is challenging because all of the defects measured from the THIs must be decomposed to characterize and quantify them. This decomposition would allow, for example, the impact of each of these defects on the performance of the prosthesis to be measured (i.e. the coefficient of friction or onset of squeaking).

Thus, the proposed study consists of measuring the evolution of the spherical surface (form, waviness and roughness) as a function of the two aforementioned phenomena (stripe wear and metal incrustation) through confocal microscopy and a modal analysis of the measurement results.

2.2.1. Characterization of spherical surface defects. The characterization of surface defects is a really important topic for researchers, but the literature provides little geometric information to quantify them on prosthetic components. Some work has already been published considering different methodologies, like multi-axis non-contact measurement method based on chromatically encoded confocal (Tuke et al 2010) or coordinate measuring machines (CMM) with uncertainty analysis and quantification (Carmignato et al 2011). However, few methods of post-processing allow a multi-scalar analysis. The method selection criteria are mainly based on the type of defects that are to be characterized (International Organization for Standardization 1998) (position, orientation, form, waviness, roughness or micro-roughness), the multi-scalar character of the method (the ability to analyse different orders of defects simultaneously) and the method's ease of implementation and use. Based on the scale of the defects that we wish to characterize, we can consider parameterizations dedicated to defect analysis on the elementary spherical geometries or more general parameterizations dedicated to a geometric analysis in which the reference element is a plane.

To describe elementary spherical geometry defects, Kanada (1995) proposes a method based on spherical harmonics to describe the defect of sphericity (form defect). This method is also used in the field of imaging and threedimensional reconstruction. Kautz et al (2002) use this approach to reduce the number of parameters corresponding to the light source in the calculation of the bidirectional reflectance distribution function. In the optics field, surface defects recurring on optical elements can be defined as a linear combination of Zernike polynomials (International Organization for Standardization 2007). Closer to the work presented in this paper, Favrelière et al (2010) characterize the form defect of a spherical surface to describe the modal deformations of spherical elements.

To characterize the surface beyond the form defect (waviness, roughness or micro-roughness), many authors use more generic methods that are not specifically dedicated to the defects of spherical geometry elements. Huang and Ceglarek (2002) propose to use a database of descriptors based on the discrete cosine transform (DCT) to analyse the different forms of a part. Another popular method is the discrete Fourier transform (DFT); in particular, the standard ISO-12781-2 (International Organization for Standardization 2011) refers to parameters involving the harmonic components of the DFT to evaluate the flatness of a surface.

The multi-scale analysis of a machined surface can be particularly useful for describing the quality of the manufactured surface, its anomalies (metallic incrustations for this study) and the resulting functional aspects (Chen and Raja 1995). In this context, different parameterizations can be used. Rosenboom et al (2011) show the manner in which a surface can be characterized and defects of very small amplitude can be detected using wavelet analysis, which allows one-dimensional (linear) or multidimensional (surface) signals to be characterized at different scales. Le Goïc et al (2011) proposes to characterize the form, waviness and roughness components of a surface using discrete modal decomposition (DMD) in the context of analysing the quality of the appearance of a surface and detecting visual anomalies thereon. DMD allows a surface to be described at different scales using a very small number of parameters. In the remainder of this paper, we develop this approach through the analysis of the contact surfaces of a THI's joint components.

2.2.2. Modal decomposition. DMD is adaptable to any type of geometry (profile or surface) and allows the defects of form, waviness and surface roughness to be characterized simultaneously (Formosa et al 2007). For this study, this method is used to quantify and compare the wear of different prostheses, regardless of the nature and extent of the wear. DMD decomposes a signal into a family of discrete functions defined a priori in a similar manner as the DFT 


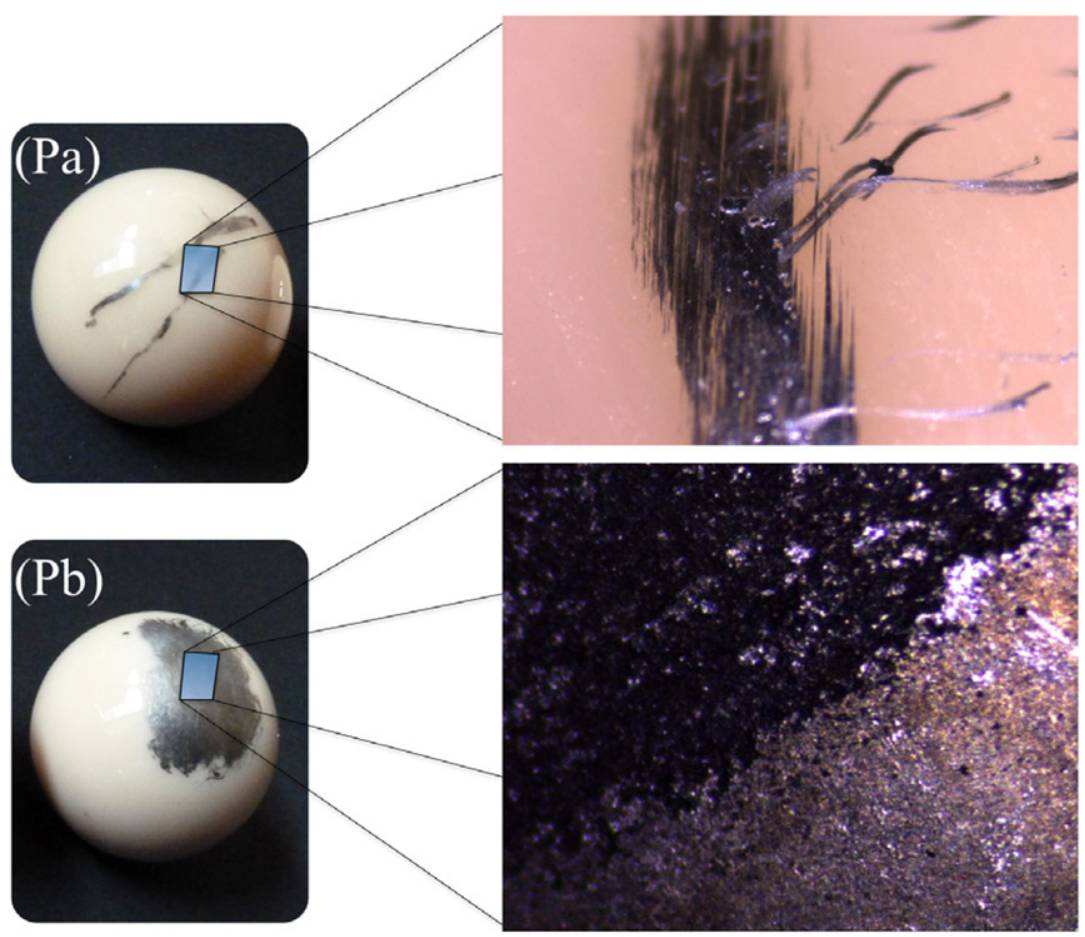

Figure 2. Sample study: explants of prosthetic heads (provided by the Tural company) .

or discrete wavelet transform. In this decomposition, the signal corresponds to the measured surface and can be a flat, cylindrical or spherical surface. More precisely, the DMD allows the surface to be decomposed into a family of descriptors (discrete functions) called modes or modal deformations. This family of functions is determined from the reference geometric element's vibration modes, which come from the solution of equation (3). For example, these functions correspond to the vibration modes of a plane if the measured surface is flat; this ability of the method is denoted by the name chosen for this parameterization (modal parameterization) (Favrelière 2009).

The decomposition operation consists of creating a projection vector (1) of measure $V$ (in vector form) in the vector space $(Q)$ formed by the vibration modes (vectors $Q_{i}$ ), as shown below:

$$
Q^{* T} \cdot V=\left(\left(Q^{T} \cdot Q\right)^{-1} \cdot Q^{T}\right) \cdot V=\lambda,
$$

where the resulting vector $\lambda$ corresponds to the contributions $\lambda_{i}$ of each mode, referred to as the modal coordinates or modal coefficients. Because the projection is performed in the non-orthonormal basis vector $(Q)$, the dual basis $Q i^{*}$ must be used. The vectors $(Q i)$ are normalized with the infinity norm $\left(\|Q i\|_{\infty}=1\right)$ to allow the contributions $\lambda_{i}$ to be obtained in metric values that are homogeneous with the measured surface. We can then express the measured surface as follows:

$$
V=\sum_{i=1}^{N_{q}} \lambda_{i} Q_{i}+\epsilon\left(N_{q}\right)
$$

where $N_{q}$ represents the number of modes selected for the decomposition and $\epsilon$ is the residual of decomposition. Modes
$Q_{i}$ are obtained from solving the following classical problem of mechanical vibration:

$K \cdot \ddot{q}+M \cdot q=0$ with $\begin{cases}M: & \text { generalized mass matrix } \\ K: & \text { generalized stiffness matrix (3) } \\ q: & \text { displacement vector. }\end{cases}$

This dynamic problem (3) can be solved either analytically (see Leissa 1969) or numerically by the finite element method (see Zienkiewicz and Taylor 2002). The problem is usually solved numerically, which provides a DMD.

Compared with the Fourier transform, the modal parameterization operates similarly but it enables any type of surface to be analysed, without a priori knowledge. Moreover, the proposed method can be adapted to more complex decompositions by inserting pre-defined forms (namely the 'technological modes') in the vector basis.

\section{Multi-scalar analysis of a spherical surface: the prosthetic head}

\subsection{Samples}

This study only uses samples of prosthetic heads that exhibit abnormalities (i.e. stripe wear or metal incrustations, see 2.1.3 and 2.1.4). Identical processes are carried out for both parts of the ball-and-socket joint (the prosthetic head and insert). To apply this method, two samples with different levels of metal incrustations were chosen (figure 2):

- prosthetic head A $(\mathrm{Pa})$, which is composed of strong ceramic BIOLOX ${ }^{\circledR}$ forte: thin metal incrustations;

- prosthetic head $\mathrm{B}(\mathrm{Pb})$, which is composed of strong ceramic BIOLOX ${ }^{\circledR}$ forte: larger metal incrustations with a zone in which deterioration appears to be more advanced. 


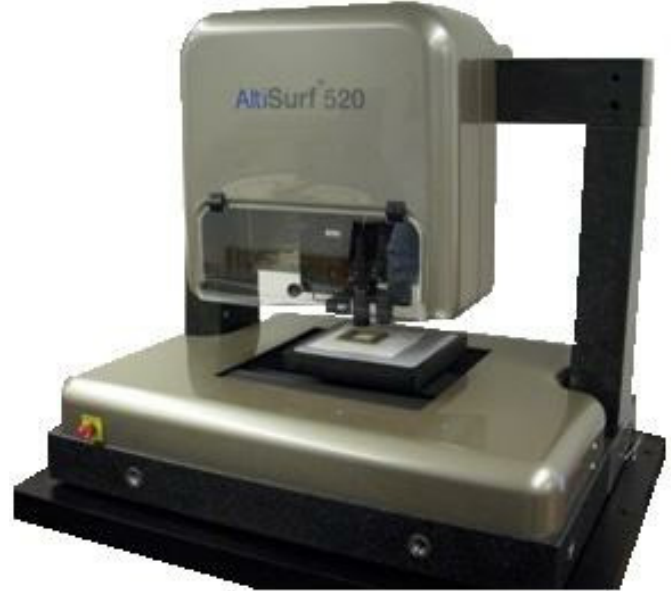

(a) Altisurf 520 instrument

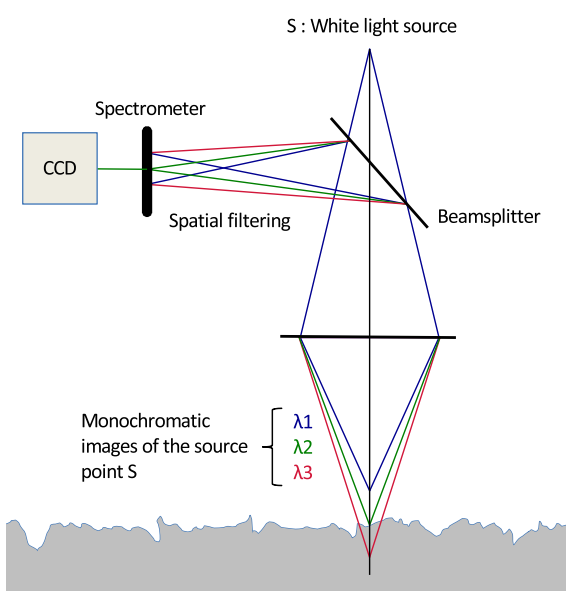

(b) Confocal microscopy method

Figure 3. Measurement method.
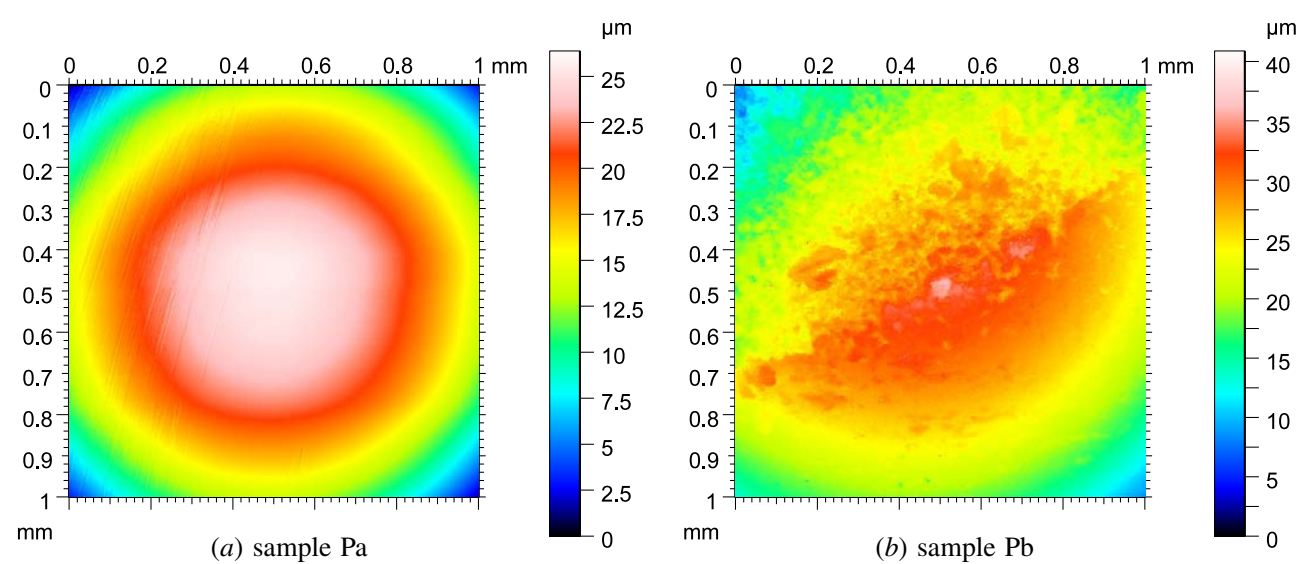

Figure 4. Representation of the measured surfaces for two samples.

\subsection{Measurement protocol}

Many optical methods, such as techniques based on interferometry, laser triangulation or confocal microscopy, permit surface topography to be precisely measured. This study uses the Altisurf 520 instrument (figure 3(a)) to obtain the measurements; this instrument is based on extended-field confocal microscopy (figure 3 (b)) (Udupa et al 2000a, 2000b). It is equipped with a chromatic confocal probe with a vertical measurement range of $300 \mu \mathrm{m}$, a vertical accuracy in $Z$ of $0.06 \mu \mathrm{m}$ and a lateral resolution of $1.55 \mu \mathrm{m}$. This non-contact measurement has already been used to measure prosthetic implants such as in Tuke et al (2010).

This study performs measurements on areas with abnormalities (figure 4) to evaluate the geometric variations of form, waviness and roughness corresponding to incrustations (figure 2). The measured surfaces are $1 \mathrm{~mm}$ squares composed of 250000 measurement points (one point every $2 \mu \mathrm{m}$ ).

\subsection{Multi-scalar analysis by the modal method}

This section describes a multi-scalar surface analysis using the modal method on the measured surfaces. The modal method algorithms are developed on Matlab ${ }^{\circledR}$, and Mountains ${ }^{\circledR}$ software is used to plot the surface topography.
3.3.1. Preamble. The objective of this study is to characterize the geometric variations of the measured surface on the joint components of hip prostheses. The nominal (reference) surfaces of prosthetic heads are spherical. The first step of this analysis is thus to filter the spherical component of the surface by calculating the associated sphere using non-deteriorated areas. The calculation of the radius and position of the centre of the best-fitted sphere minimizes the distance between the surface and a sphere according to the criterion of least-squares.

Figure 5 shows the resulting surfaces. The form, waviness and roughness defects will be subsequently characterized from this initial step.

3.3.2. Implementation of the modal method. DMD is the central step of the modal method (section 2.2). As a reminder, this step decomposes the surface ${ }^{\text {meas }} V$ into a family of descriptors derived from the mechanical vibration. These descriptors correspond to the natural vibration modes of the reference geometry, which here is a plane (figure 6) after the sphere is filtered through the least-squares method. This family of descriptors forms a vector space, referred to as a modal basis. 


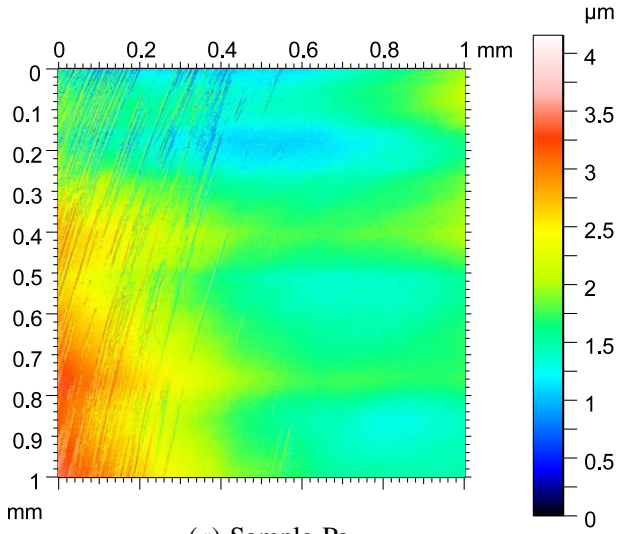

(a) Sample Pa

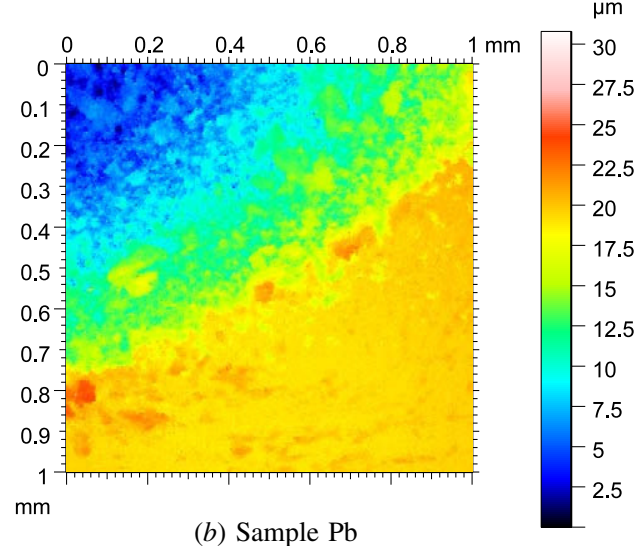

(b) Sample $\mathrm{Pb}$

Figure 5. Reconstruction of the surfaces obtained after the removal of the original form (sphere) by the least-squares criterion.
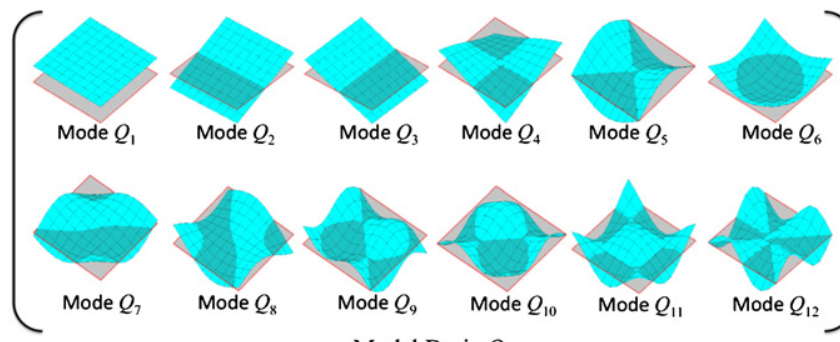

Modal Basis $Q$

Figure 6. First six natural vibration modes of a flat surface

After projecting the measured surface in the modal basis, the decomposition is obtained (4). For this case, we set the number of modes $\left(N_{q}\right)$ equal to 500 for the decomposition:

$$
{ }^{\text {meas }} V=\sum_{i=1}^{N_{q}=500} \lambda_{i} Q_{i}+\epsilon\left(N_{q}=500\right) \text {, }
$$

where the vector $\lambda$ corresponds to the modal contribution $\lambda_{i}$ of each mode and $Q_{i}$ to the vectors of the plane modal basis. The DMD can be visualized in the form of a modal amplitude spectrum. Figure 7 shows the spectrum with the 500 modes of the decomposition.

Note that the modal spectrum of the decomposition of this surface is contained in a very rapidly decreasing double envelope. A negative amplitude on the modal spectrum represents the corresponding opposite mode. Modes $\left(Q_{i}\right)$ are sorted in order of frequency, but the form of the envelope shows that they are simultaneously sorted in order of amplitude. This feature allows the DMD results to be used to reconstruct the form, waviness and roughness defects of the decomposed surfaces. Furthermore, the shape of the spectrum (rapid decrease) indicates that a small number of parameters can effectively represent the surface.

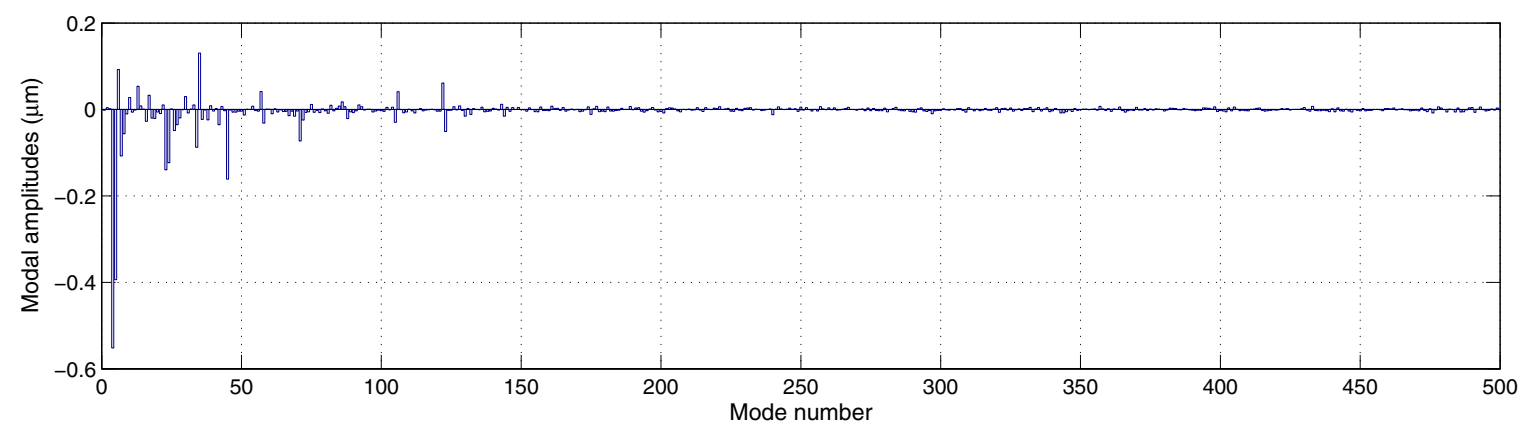

(a) sample $\mathrm{Pa}$

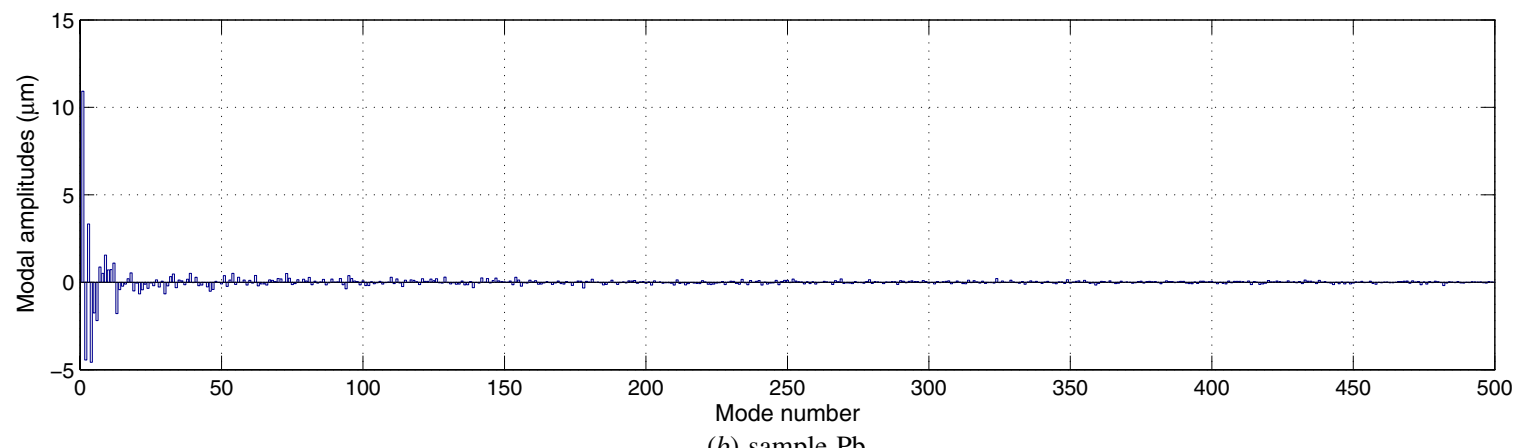

(b) sample $\mathrm{Pb}$

Figure 7. Representation of the modal amplitude spectrum. 


\subsubsection{Modal filtering.}

(a) Residual. The residual, denoted by $\epsilon$, is used to compare the measured surface and the surface reconstructed from all of the decomposition modes. This step allows us to evaluate the relevance of the modal parameters and the quality of the approximation made for the surface considered. The residual of the DMD is obtained by

$$
\epsilon\left(N_{w}\right)={ }^{\text {meas }} V-\sum_{i=1}^{N_{w}} \lambda_{i} Q_{i} .
$$

Figure 8 shows the residual of the modal decomposition for sample Pa.

Note that this residual is of very low amplitude $(<1.8 \mu \mathrm{m})$ and contains high amplitudes from the threshold $N_{w}+1$ (i.e. very low periodicity) but also aperiodic components and measurement noise. This measurement noise has already been evaluated and decreased in a previous work with a calibration protocol (Favreliere et al 2011). With this calibration protocol, the systematic geometrical errors related to the mechanical guiding system are corrected and the final measurement noise amplitude is decreased to $0.5 \mu \mathrm{m}$ for all the measurement range $(200 \mathrm{~mm} \times 200 \mathrm{~mm})$. For the two studied samples $(1 \mathrm{~mm} \times 1 \mathrm{~mm})$, we assume that the measurement noise is lower and significantly equal to the optical sensor noise $(0.06 \mu \mathrm{m})$. Modal decomposition therefore provides a very good approximation of the surface from a set of measured data points.

(b) Form, waviness and roughness defects. The modal method can reconstruct the different components of a surface by reconstructing parts of the DMD. The form, waviness and roughness defects of a surface can then be obtained by the simple reconstruction of a part of the modal amplitude spectrum (figure 9).

The form, waviness and roughness components are obtained by reconstructing part of the modal spectrum according to the following equations (6):

$$
\left\{\begin{array}{l}
{ }^{\text {form }} V=\sum_{i=1}^{N_{f}} \lambda_{i} Q_{i} \\
\text { waviness } V=\sum_{i=N_{f}+1}^{N_{w}} \lambda_{i} Q_{i} \\
\text { roughness } V={ }^{\text {meas }} V-\sum_{i=1}^{N_{w}} \lambda_{i} Q_{i} .
\end{array}\right.
$$

The choice of the two thresholds, form/waviness $\left(N_{f}\right)$ and waviness/roughness $\left(N_{w}\right)$, influences the way in which the defect types are classified (figure 9). The standards do not clearly define how to set these thresholds, and our studies do not provide additional information about the thresholds. However, DMD users can easily vary these thresholds, according to the visualization of reconstructed surfaces, these thresholds can be defined. To illustrate the method, we can see in figure 10 some examples of the reconstruction of the surface $\mathrm{Pb}$ for several selections of number of modes (variation of $N_{f}$ ).

Thus, for each analysed object, users can define what they see as being in the order of a defect for the form, waviness or roughness. Figures 11 and 12 show the reconstructions of the form, waviness and roughness components obtained by the modal method for both surfaces studied.

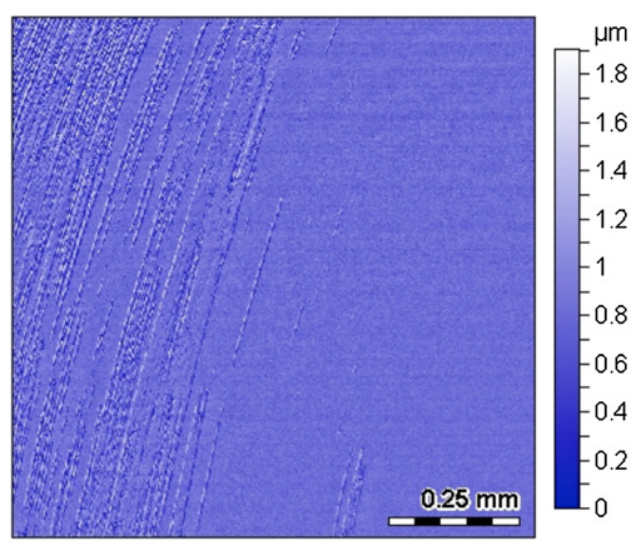

Figure 8. Representation of the residual of the DMD calculated on the surface $(\mathrm{Pa})$.

\section{Influence of sphere filtering}

The same studies of modal filtering are here conducted on measured surfaces without a previous best fitting of the sphere. Figures 13 and 14 show the reconstructions of the form, waviness and roughness components obtained by the modal method for both the studied surfaces without the best fitting of the sphere.

The presented results show that form and waviness components are affected by the initial spherical form. However, the roughness component seems unchanged. In order to compare the two approaches, we propose to compute the modal decomposition of a nominal sphere, with radius equal to $14 \mathrm{~mm}$, to determine the impact of the initial spherical form in the modal decomposition. The reconstruction results (see figure 15) showed that the nominal sphere is well reconstructed in the form component $\left(N_{f}=40\right)$. Part of the reconstruction on the waviness component $\left(N_{w}=150\right)$, with an amplitude of $0.2 \mu \mathrm{m}$, remains to be done. But we can observe that the amplitude of the roughness component is very low, $0.05 \mu \mathrm{m}$ or $0.3 \%$ of the initial amplitude. We can therefore conclude that the two filtering approaches of the initial spherical form do not affect the roughness component.

\section{Using the results}

The multi-scale analysis allows a surface to be decomposed in terms of three main parameters: form, waviness and roughness defects. From the two prosthetic heads measured in this study, we have purposefully chosen two explants with wears that are not of the same type to demonstrate the usefulness of the proposed method. The wear on the head of $\mathrm{Pa}$ is thin and 'superficial' (surface deterioration), while that on the head of $\mathrm{Pb}$ has greater deterioration, with pronounced roughness and chipping that is visible to the naked eye (figure 2). Thus, of the chosen samples, the identification of different surface components will help to quantify the form, waviness and roughness components of both surfaces despite their very different typology.

The proposed method allows the simultaneous analysis and identification of different orders of defects on a surface 


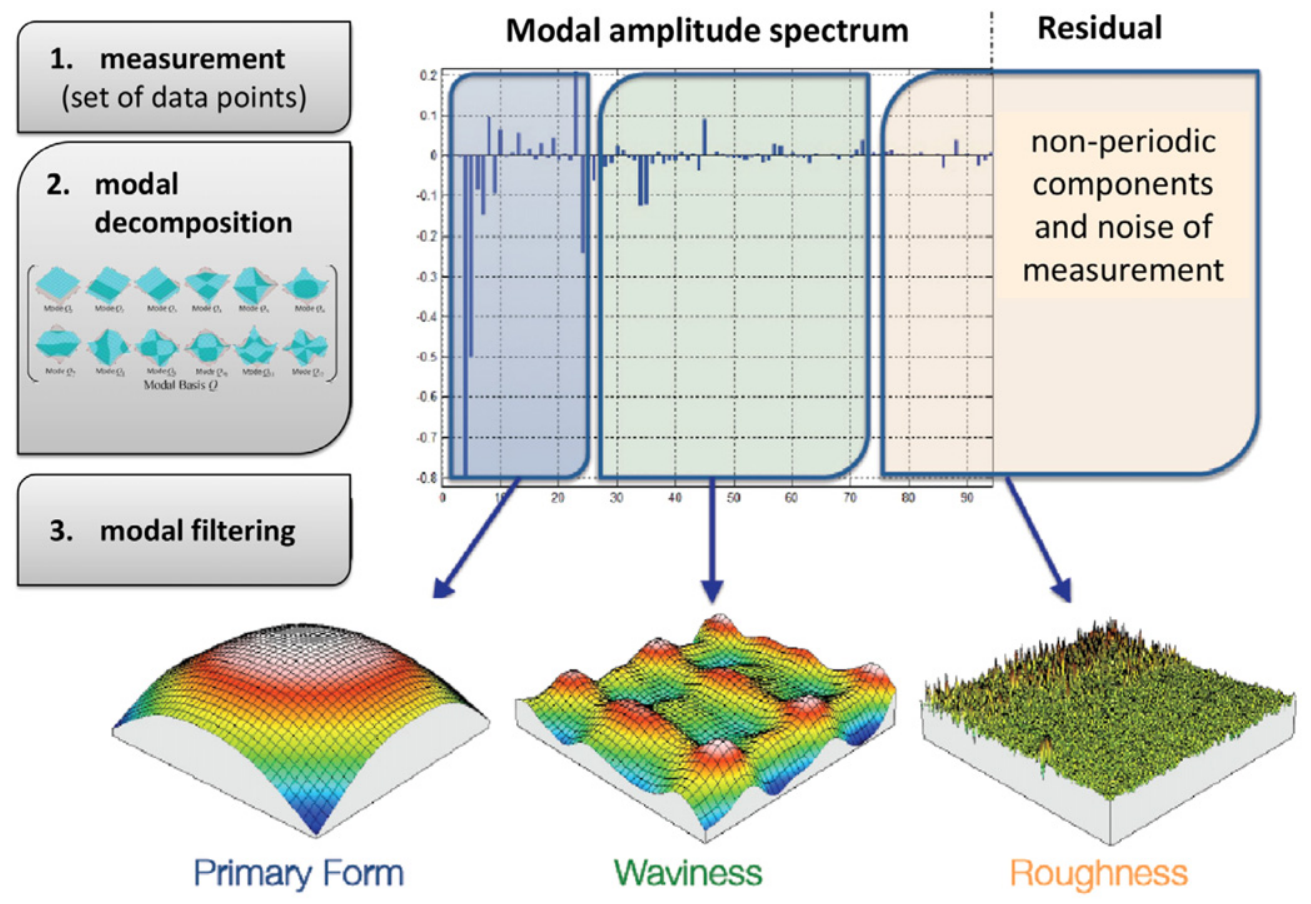

Figure 9. Steps of the modal method.
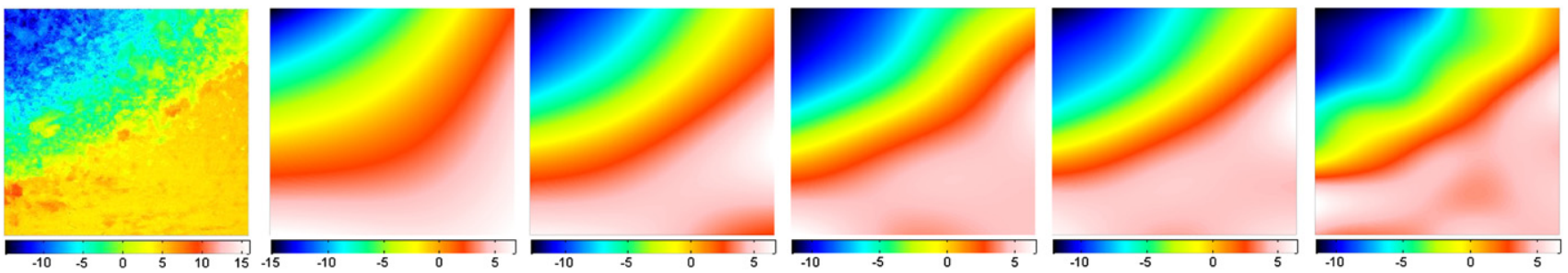

Figure 10. Surface $\mathrm{Pb}$ and the form reconstruction with $N_{f}=5,10,15,20$ and 40 .
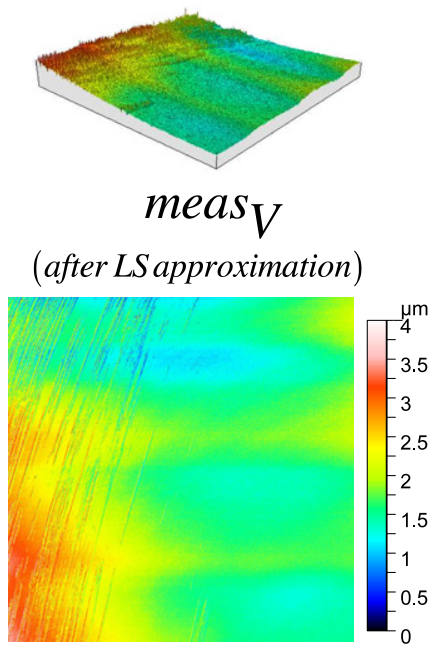

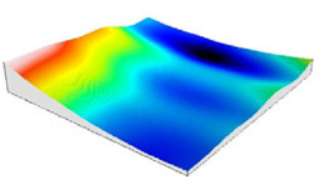

form $_{V}=\sum_{i=1}^{n f} \lambda_{i} Q_{i}$

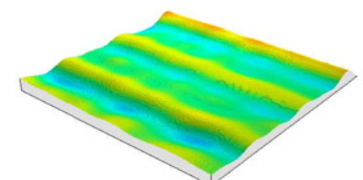

${ }_{\text {waviness }} V=\sum_{i=n f}^{n w} \lambda_{i} Q_{i}$

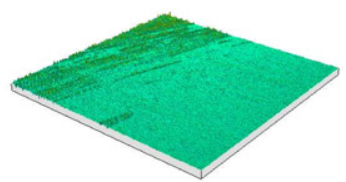

${ }^{\text {roughness }} V={ }^{\text {meas }} V-\sum_{i=1}^{n w} \lambda_{i} Q_{i}$
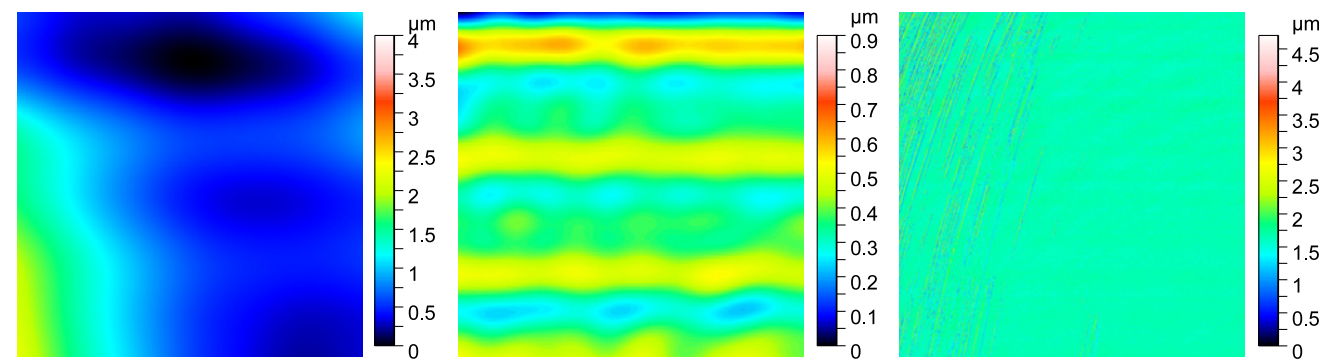

Figure 11. Multi-scale analysis of the prosthetic head Pa, with $N_{f}=40$ and $N_{w}=150$.

without a priori knowledge of the surface's form or other characteristics. For example, there is a ten-fold difference between the form defects of samples $\mathrm{Pa}$ and $\mathrm{Pb}$ due to the chipping of prosthesis $\mathrm{Pb}$ (figures 11 and 12), confirming the difference already visible to the naked eye. This result is also visible in the spectrum (figure 7) when comparing the contributions of the form modes.

Similarly, the method allows the roughness defects between the two chosen samples to be identified and compared. The method illustrates the advantage of the modal method for 


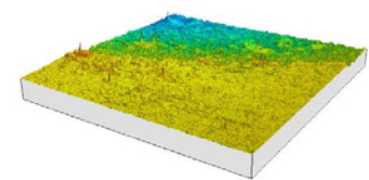

meas $_{V}$

(after LSapproximation)

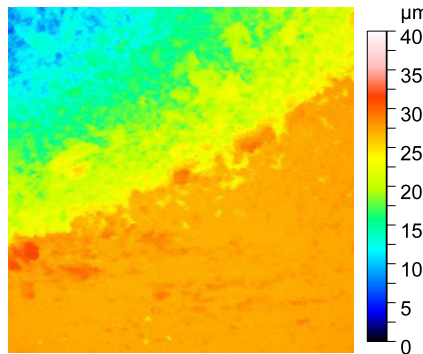

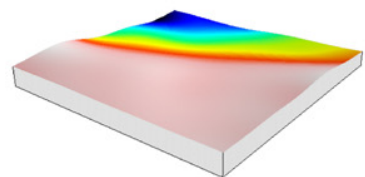

form $_{V}=\sum_{i=1}^{n f} \lambda_{i} Q_{i}$

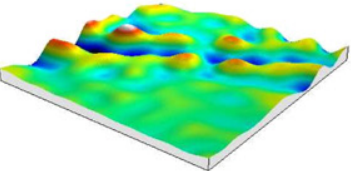

${ }^{\text {waviness }} V=\sum_{i=n f}^{n w} \lambda_{i} Q_{i}$

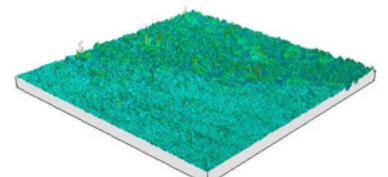

${ }^{\text {roughness }} V={ }^{\text {meas }} V-\sum_{i=1}^{n w} \lambda_{i} Q_{i}$
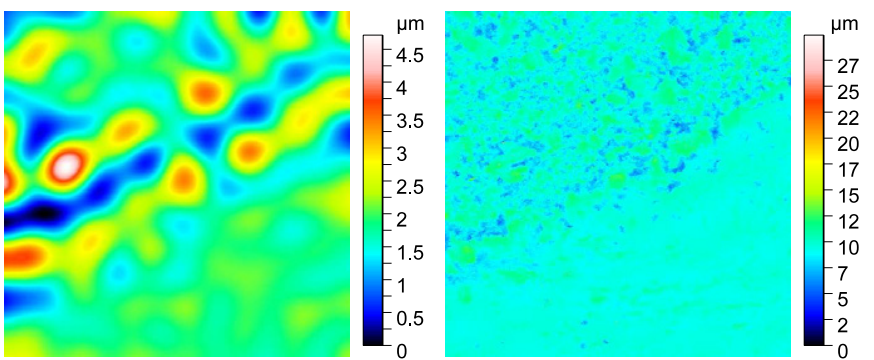

Figure 12. Multi-scale analysis of the prosthetic head Pb, with $N_{f}=40$ and $N_{w}=150$.
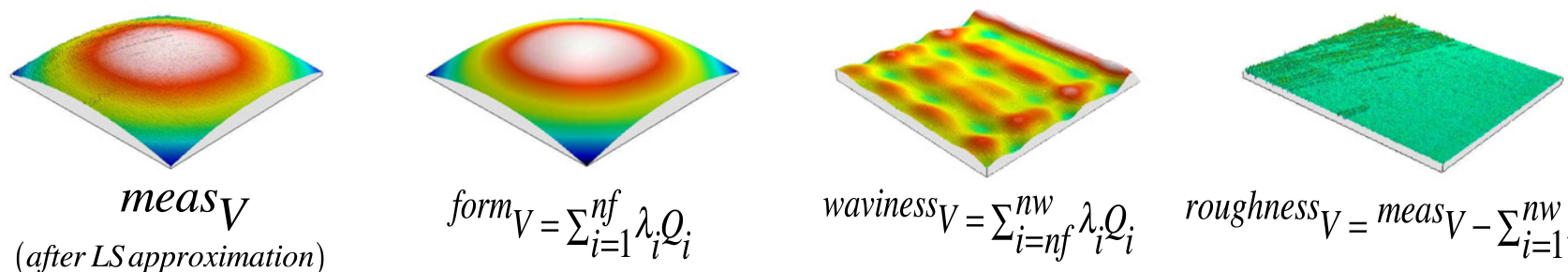

${ }_{\text {waviness }} V=\sum_{i=n f}^{n w} \lambda_{i} Q_{i}$

${ }^{\text {roughness }} V={ }^{\text {meas }} V-\sum_{i=1}^{n w} \lambda_{i} Q_{i}$
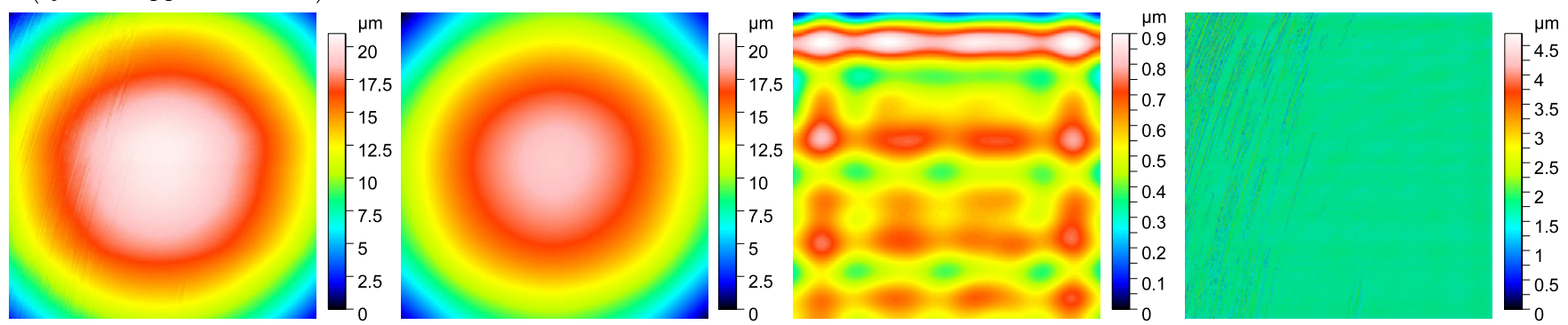

Figure 13. Multi-scale analysis of the prosthetic head Pa without sphere fitting, with $N_{f}=40$ and $N_{w}=150$.

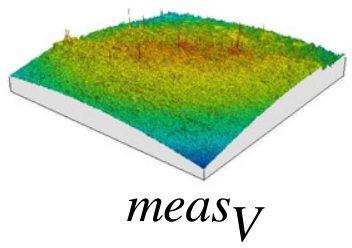

(after LS approximation)
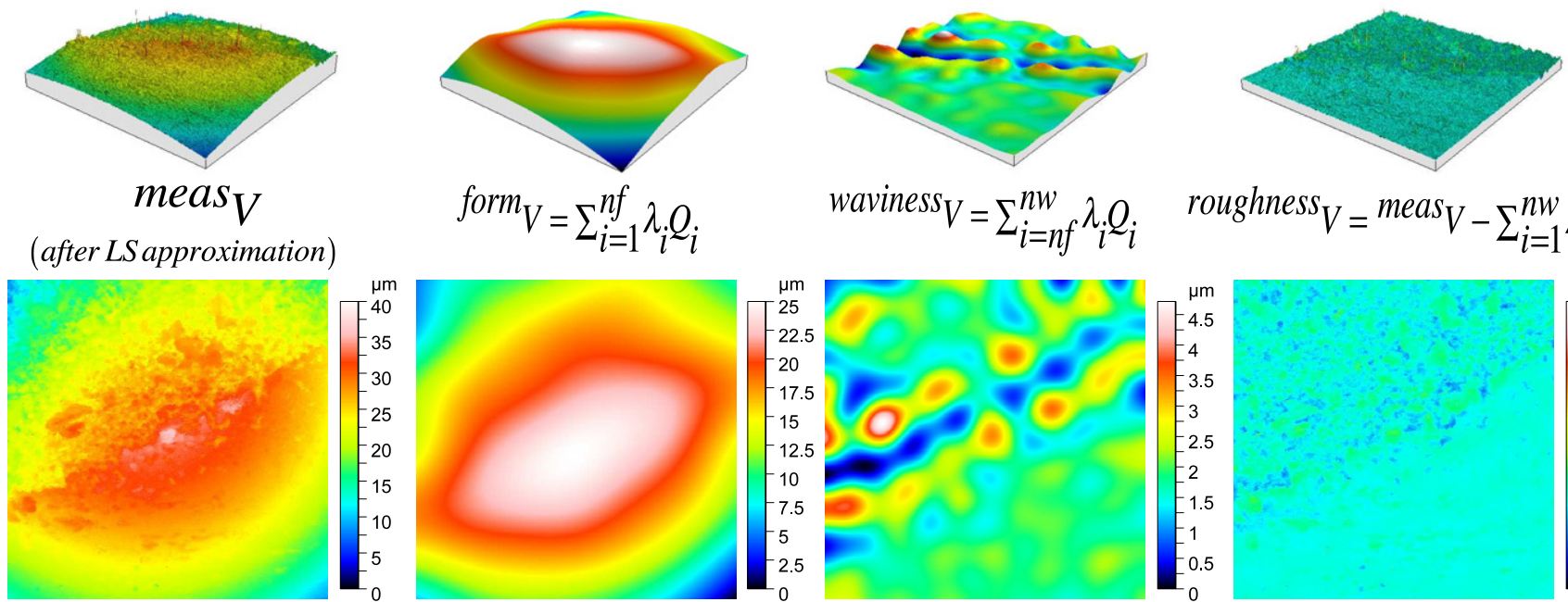

${ }^{\text {waviness }} V=\sum_{i=n f}^{n w} \lambda_{i} Q_{i}$

${ }^{\text {roughness }} V={ }^{\text {meas }} V-\sum_{i=1}^{n w} \lambda_{i} Q_{i}$
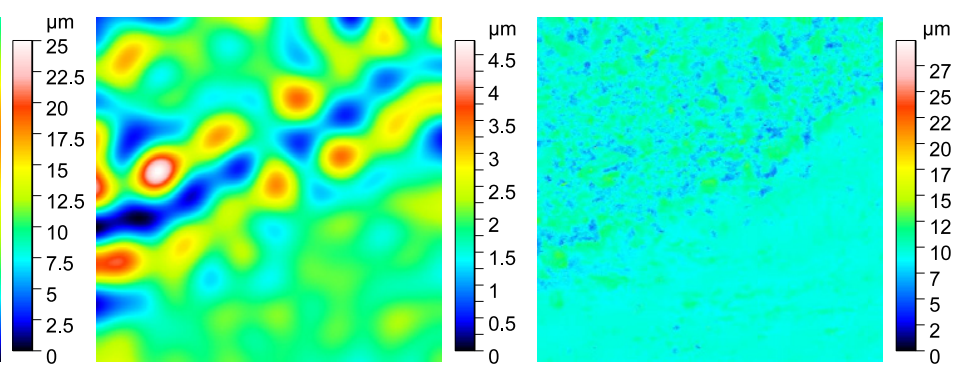

Figure 14. Multi-scale analysis of the prosthetic head $\mathrm{Pb}$ without sphere fitting, with $N_{f}=40$ and $N_{w}=150$. 


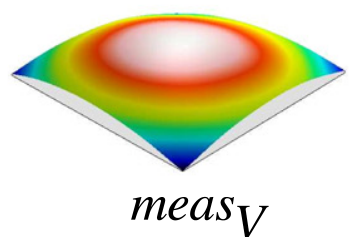

(after LS approximation)

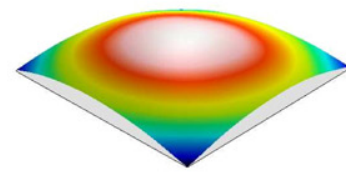

form $_{V}=\sum_{i=1}^{n f} \lambda_{i} Q_{i}$

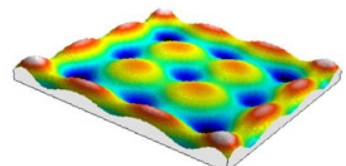

${ }^{\text {waviness }} V=\sum_{i=n f}^{n w} \lambda_{i} Q_{i}$

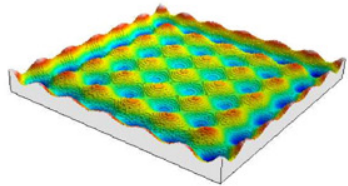

${ }^{\text {roughness }} V={ }^{\text {meas }} V-\sum_{i=1}^{n w} \lambda_{i} Q_{i}$
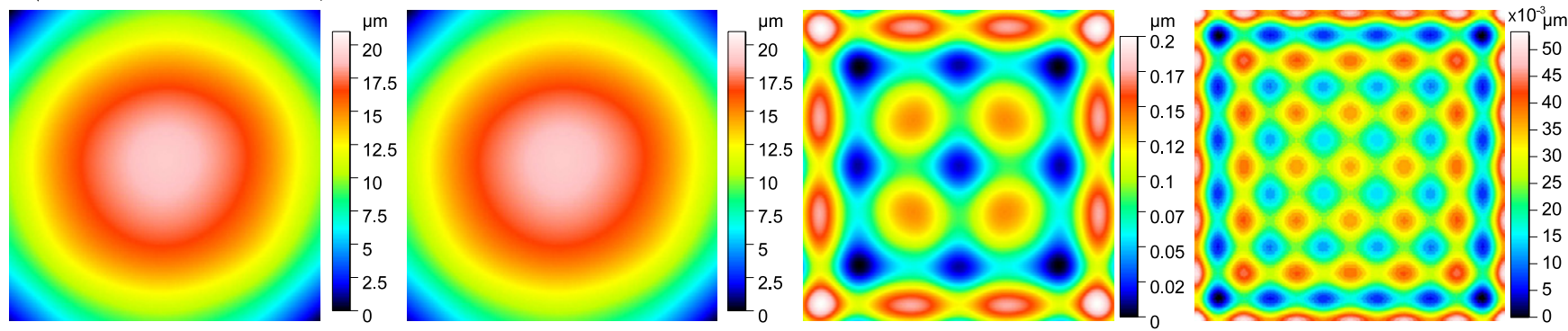

Figure 15. Multi-scale analysis of a theoretical sphere, with $N_{f}=40$ and $N_{w}=150$.

identifying the various orders of defects of a surface in a single analysis without knowing a priori on which surface component the deterioration exists.

We show in particular that the modal method can extract roughness defects of the order of the roughness (high frequencies) with amplitudes greater than those of the form and waviness defects. This finding is visible in figure 11, in which the roughness has an amplitude of approximately $1.6 \mu \mathrm{m}$ and the waviness has an amplitude of approximately $0.7 \mu \mathrm{m}$. This finding also applies to prosthesis $\mathrm{Pb}$ (figure 12), for which the amplitudes of the roughness and waviness are approximately $17 \mu \mathrm{m}$ and $4 \mu \mathrm{m}$, respectively. Thus, the modal method allows the different surface components to be extracted and compared regardless of the surface types.

Finally, the filtering quality for form and waviness performed on the surfaces allows the stripes' amplitude to be precisely measured and enables us to see that on the surface of $\mathrm{Pa}$, the stripes have a primary direction of propagation that follows the direction of the incrustation mark. It can also be noted that these stripes have a thickness that exceeds the nominal surface (figure 8), confirming the metallic deposit hypothesis. However, some of the stripes are below the nominal diameter. We can thus assume that third-body particles have already scratched the surface of the head before becoming incrusted. However, the particles can also originate from a small deposit left by the surgeon during the implantation of the prosthesis due to friction between the tools and ceramic parts that has amplified over time. The advanced incrustation of the $\mathrm{Pb}$ prosthesis has a roughness of a completely different type because roughness is more homogeneous and has an amplitude of approximately $17 \mu \mathrm{m}$ (figure 12). The width of the incrustation bands is similar to that of the strip wear bands, which suggests that the roughness due to stripe wear allowed the incrustation of third-body particles in the prosthesis.

\section{Conclusion and perspectives}

The purpose of this study is to provide elements of analysis to help better understand the phenomena of metal incrustations on hip joint components. With this in mind, we develop a metrological approach to this phenomenon to determine the consequences that these incrustations have on the surface condition (roughness) and the geometric variations in terms of form and waviness defects.

We develop an innovative surface analysis and filtering method referred to as the modal method. From measurements made on the contact areas of the joint components of hip prostheses, the modal method allows different orders of geometric defects to be identified from the surfaces studied. We show that the DMD allows an accurate multi-scale analysis of surfaces with different types of defects. We show that it is possible that the metal marks visible on the prosthetic head may have provoked either more or less advanced deterioration of the prosthesis surface. While the marks on the sample $\mathrm{Pa}$ did not degrade the surface in more than a superficial manner (scratches on the order of the roughness or modification of the surface condition), the more 'marked' prosthesis sample $\mathrm{Pb}$ affected all of the geometrical surface components, resulting in a major form defect.

The distinction and characterization of the different types of defects by the DMD can be useful for classifying different prostheses or establishing a defect database. Thus, depending on the types of prostheses and conditions of use, a defect database can be established based on the distribution of the modal amplitude spectrum coefficients.

A subsequent study will consist of using this method on in vitro tests to measure the influence of geometric variations on the dysfunction of hip prostheses with these abnormalities (coefficient of friction, onset of squeaking, among others). More specifically, this future study will compare the components of deteriorated prostheses with those of new prostheses, as well as prostheses before and after a wear test. Discrete mode decomposition can also generate these defects on the numerical modelling of the prosthesis based on the modal amplitude spectrum. This approach also allows the selection of the types of defects to be modelled. Thus, one can consider coupling the in vitro tests with numerical simulations to develop analytical models of behaviour. 


\section{Acknowledgments}

This research effort is supported by the research program IDEFORA. These works have been done in collaboration between the Symme Lab (Annecy) and I2M Lab (Bordeaux), and applied to samples of the Tural Company, partner of the program.

\section{References}

Carmignato S, Spinelli M, Affatato S and Savio E 2011 Uncertainty evaluation of volumetric wear assessment from coordinate measurements of ceramic hip joint prostheses Wear 270 584-90

Chen X, Raja J and Simanapalli S 1995 Multi-scale analysis of engineering surfaces Int. J. Mach. Tools Manuf. 35 231-8

Chevillotte C, Trousdale R, Guyen O, Chen Q and Berry D 2008 Etude expérimentale des phénomènes de grincement ou 'squeaking' avec les couples de frottement céramique/céramique dans les prothèses totales de hanche Journées Lyonnaises de Chirurgie de la Hanche pp 1-4

Favrelière H 2009 Modal tolerancing: from metrology to specifications $P h D$ Thesis Université de Savoie

Favreliere H, Goic G, Samper S and Belin P 2011 Flatness calibration of $x y$ translation stages through modal parameterization Proc. 15th Int. Congress of Metrology (Paris)

Favrelière H, Samper S and Adragna P-A 2010 Caractérisation des défauts d'une surface sphérique par décomposition modale arXiv: 1002.0251

Formosa F, Samper S and Perpoli I 2007 Modal expression of form defects Models for Computer Aided Tolerancing in Design and Manufacturing ed J K Davidson (Berlin: Springer) pp 13-22

Hannouche D, Nich C, Bizot P, Meunier A, Nizard R and Sedel L 2003 Fractures of ceramic bearings: history and present status Clin. Orthop. Relat. Res. 417 19-26

Huang W and Ceglarek D 2002 Mode-based decomposition of part form error by discrete-cosine-transform with implementation to assembly and stamping system with compliant parts $C I R P$ Ann. Manuf. Technol. 51 21-6

International Organization for Standardization 2007 Optics and photonics - preparation of drawings for optical elements and systemspart 5: surface form tolerances ISO-10110-5

International Organization for Standardization 2011 Geometrical product specifications (GPS) - flatness-part 2: specification operators ISO-12781-2

International Organization for Standardization 1998 Geometrical product specification (GPS)—surface imperfections-terms, definitions and parameters $I S O-8785$

Jarrett C A, Ranawat A S, Bruzzone M, Blum Y C, Rodriguez J A and Ranawat C S 2009 The squeaking hip: a phenomenon of ceramic-on-ceramic total hip arthroplasty J. Bone Joint Surgery 91 1344-9

Kanada T 1995 Evaluation of spherical form errors-computation of sphericity by means of minimum zone method and some examinations with using simulated data Precis. Eng. 17 281-9
Kautz J, Sloan P and Snyder J 2002 Fast, arbitrary BRDF shading for low-frequency lighting using spherical harmonics Proc. 13th Eurographics Workshop on Rendering (Pisa, Italy) (Aire-la-Ville, Switzerland: Eurographics Association) pp 291-6

Le Goïc G, Favrelière H, Samper S and Formosa F 2011 Multi scale modal decomposition of primary form, waviness and roughness of surfaces Scanning 33 332-41

Leissa A W 1969 Vibration of Plates (NASA SP-160) pp 1-316

Matar W Y, Restrepo C, Parvizi J, Kurtz S M and Hozack W J 2010 Revision hip arthroplasty for ceramic-on-ceramic squeaking hips does not compromise the results J. Arthroplasty 25 81-86

Nevelos J, Ingham E, Doyle C, Streicher R, Nevelos A, Walter W, Fisher J and Eng D 2000 Microseparation of the centers of alumina-alumina artificial hip joints during simulator testing produces clinically relevant wear rates and patterns J. Arthroplasty 15 793-5

Restrepo C, Parvizi J, Kurtz S, Sharkey P, Hozack W and Rothman R 2008 The noisy ceramic hip: is component malpositioning the cause? J. Arthroplasty 23 643-9

Restrepo C, Post Z, Kai B and Hozack W 2010 The effect of stem design on the prevalence of squeaking following ceramic-on-ceramic bearing total hip arthroplasty J. Bone Joint Surg. 92 550-7

Rosenboom L, Kreis T and Jüptner W 2011 Surface description and defect detection by wavelet analysis Meas. Sci. Technol. 22045102

Sariali E, Stewart T, Jin Z and Fisher J 2010 Three-dimensional modeling of in vitro hip kinematics under micro-separation regime for ceramic on ceramic total hip prosthesis. An analysis of vibration and noise J. Biomech. 43 326-33

Swanson T V, Peterson D J, Seethala R, Bliss R L and Spellmon C A 2010 Influence of prosthetic design on squeaking after ceramic-on-ceramic total hip arthroplasty J. Arthroplasty 25 36-42

Taylor S, Manley M T and Sutton K 2007 The role of stripe wear in causing acoustic emissions from alumina ceramic-on-ceramic bearings $J$. Arthroplasty 22 47-51

Tuke M, Taylor A, Roques A and Maul C 2010 3D linear and volumetric wear measurement on artificial hip joints-validation of a new methodology Precis. Eng. 34 777-83

Udupa G, Singaperumal M, Sirohi R and Kothiyal M P 2000a Characterization of surface topography by confocal microscopy: I. Principles and the measurement system Meas. Sci. Technol. 11 305-14

Udupa G, Singaperumal M, Sirohi R and Kothiyal M 2000b Characterization of surface topography by confocal microscopy: II. The micro and macro surface irregularities Meas. Sci. Technol. 11 315-29

Walter W L, Yeung E and Esposito C 2010 A review of squeaking hips Orthop. Adv. 18 1-8

Zienkiewicz O and Taylor R 2002 The Finite Element Method for Solid and Structural Mechanics 6th edn (Oxford: Butterworth-Heinemann) ISBN 0-7506-6320-0 